\title{
Mobilidade de oxigênio intersticial em cerâmicas $\mathrm{SmBa}_{2} \mathrm{Cu}_{3} \mathrm{O}_{7-\delta}$
}

\section{(Mobility of interstitial oxygen in $\mathrm{SmBa}_{2} \mathrm{Cu}_{3} \mathrm{O}_{7-\delta}$ ceramics)}

\author{
R. M. Nascimento , C. R. Grandini ${ }^{1}$, J. M. A. Gimenez ${ }^{1}$, A. G. da Cunha ${ }^{2}$ \\ ${ }^{1}$ Grupo de Relaxações Anelásticas, UNESP, Bauru, SP 17033-360 \\ ${ }^{2}$ Departamento de Física, UFES, Vitória, ES 29060-900
}

\begin{abstract}
Resumo
Desde a descoberta dos supercondutores com alta temperatura crítica, vários trabalhos têm sido efetuados sobre as diferentes propriedades destes materiais. As cerâmicas $\mathrm{YBa}_{2} \mathrm{Cu}_{3} \mathrm{O}_{7-\delta}$ mostraram que têm suas propriedades supercondutoras fortemente afetadas pela quantidade de oxigênio. Mais recentemente, medidas de relaxação anelástica em $\mathrm{La}_{2} \mathrm{CuO}_{4+\delta}$ mostraram que a remoção deste elemento pode ser relacionada com dois eventos. Um deles é o decréscimo na mobilidade entre dois planos adjacentes $\mathrm{CuO}$ e o outro, é o aumento no número de padrões de mobilidade para os octaedros $\mathrm{CuO}_{6}$. Para $\mathrm{SmBa}_{2} \mathrm{Cu}_{3} \mathrm{O}_{7}(\mathrm{SBCO})$, a possibilidade de uma estequiometria de oxigênio variável e sua alta mobilidade nos planos $\mathrm{CuO}_{\mathrm{x}}$, dá origem a um diagrama de fases bastante rico. Neste trabalho, são reportadas medidas de espectroscopia anelástica em amostras de SBCO, que mostram um pico de relaxação termicamente ativado, que foi atribuído aos saltos de átomos de oxigênio nas posições O1 e O5 da rede.
\end{abstract}

Palavras-chave: oxigênio intersticial, óxidos SBCO, cerâmicas supercondutoras.

\begin{abstract}
Since the discovery of the high-Tc superconductors, several works have been made about the different properties of these materials. The $\mathrm{YBa}_{2} \mathrm{Cu}_{3} \mathrm{O}_{7-\delta}$ ceramic showed to have its superconducting properties strongly affected by the oxygen content. More recently, anelastic relaxation measurements in $\mathrm{La}_{2} \mathrm{CuO}_{4+\delta}$, showed that the removal of this element can be related to two events. One is the decrease in mobility between two adjacent $\mathrm{CuO}$ planes, and the other is the increase in the number of tilting patterns of the $\mathrm{CuO}_{6}$ octahedra. For $\mathrm{SmBa}_{2} \mathrm{Cu}_{3} \mathrm{O}_{7}(\mathrm{SBCO})$, the possibility of variable stoichiometry and the high mobility of oxygen in the CuO $\mathrm{O}_{x}$ planes give rise to a rich phase diagram. In this paper, anelastic spectroscopy measurements are reported in SBCO samples which shown a thermally activated relaxation peak that was attributed to the jumps of the oxygen atoms in the inter-chains $O 1$ and 05 of the lattice.
\end{abstract}

Keywords: interstitial oxygen, SBCO oxides, superconductor ceramics.

\section{INTRODUÇÃO}

$\mathrm{O}$ composto $\mathrm{SmBa}_{2} \mathrm{Cu}_{3} \mathrm{O}_{7-\delta}$ (SBCO), obtido pela substituição do íon $\mathrm{Y}$ pelo $\mathrm{Sm}$ no muito bem conhecido e estudado $\mathrm{YBa}_{2} \mathrm{Cu}_{3} \mathrm{O}_{7-\delta}$ (YBCO), é potencialmente atrativo para uma melhor compreensão dos mecanismos da supercondutividade em Física e para aplicações como dispositivos eletrônicos. As amostras de SBCO possuem temperatura crítica $\left(\mathrm{T}_{c}\right)$ maior que a do YBCO $[1,2]$ e uma solubilidade maior do íon terra rara que no solvente $\mathrm{Ba}-\mathrm{Cu}$ $\mathrm{O}$ [3], fazendo com que o processo de crescimento seja mais rápido.

Oxigênio não estequiométrico (intersticial) influencia profundamente as propriedades físicas de vários óxidos, em particular, criando buracos. Isto foi imediatamente compreendido nos óxidos de $\mathrm{YBa}_{2} \mathrm{Cu}_{3} \mathrm{O}_{6+\delta}$ (estrutura 123), onde todos os buracos condutores são criados pelos átomos de oxigênio que excedem a estequiometria 6 nos planos $\mathrm{CuO}_{\mathrm{x}}$. Acredita-se, ainda, que a quantidade de buracos criados não depende somente da quantidade de oxigênio intersticial $\delta$, mas também de seu ordenamento [4]. O reordenamento dos átomos de oxigênio deve ocorrer acima da temperatura ambiente, devido a uma mobilidade alta não usual destes átomos de oxigênio.

Muitos esforços têm sido feitos para estudar a mobilidade de oxigênio nos óxidos 123 e dentre as várias técnicas utilizadas, as medidas de relaxações anelásticas são as mais adequadas, pois permitem a determinação precisa da frequiência de saltos de uma espécie atômica, independente do modelo utilizado e, se diferentes tipos de saltos atômicos são possíveis, eles podem ser seletivamente discriminados. De fato, um pico no espectro da perda da energia elástica como função da temperatura aparece a temperaturas nas quais um tipo particular de salto atômico ocorre numa taxa igual a $2 \pi$ vezes a freqüência de vibração da amostra. $\mathrm{O}$ percurso em temperatura do espectro da absorção da energia elástica permite a determinação dos processos de relaxação que ocorrem numa amostra. 
A quantidade de oxigênio é um fator crucial para determinar a supercondutividade e outras propriedades físicas do SBCO. A investigação da difusão de oxigênio ainda possui um papel importante em pesquisa fundamental e aplicada. Os supercondutores SBCO são deficientes em oxigênio $(\delta<0,7)$ como sinterizados e, como uma conseqüência, isolante. Um passo essencial em sua preparação é inserir oxigênio após a sinterização através de um recozimento ou resfriamento lento em atmosfera de oxigênio para induzir supercondutividade. A difusão de oxigênio através da rede ocorre nos planos basais entre as posições vazias $\mathrm{O} 1$ e $\mathrm{O} 5$. A incorporação de oxigênio não apresenta dificuldade em amostras convencionalmente preparadas nas quais, devido a sua alta porosidade, o oxigênio é apto a se difundir rapidamente através do volume do material [4]. A dinâmica de oxigênio pode ser estudada por intermédio de medidas de relaxação anelástica (espectroscopia anelástica), uma técnica bastante potente para diferenciar com clareza ordenamento atômico de curto alcance e/ou processos de desordem [5].

$O$ papel do oxigênio intersticial em outros supercondutores com alta temperatura crítica, onde os buracos são criados principalmente por substituição de cátions, foi menos estudado, porém, pelo menos em $\mathrm{La}_{2} \mathrm{CuO}_{4+\delta}$ (estrutura 214) é bastante similar [6]. De fato, todos os óxidos 214 são capazes de acomodar quantidades relativamente altas de oxigênio intersticial, que são bastante móveis e contribuem para dopar com buracos os planos $\mathrm{CuO}_{2}$. Em adição, oxigênio intersticial em $\mathrm{La}_{2} \mathrm{CuO}_{4+\delta}$ forma fases ordenadas e possivelmente pares ligados covalentemente, os quais contribuem menos para a dopagem com buracos $[7,8]$.

O aspecto dinâmico é investigado por métodos indiretos, por exemplo, a partir da dependência das propriedades

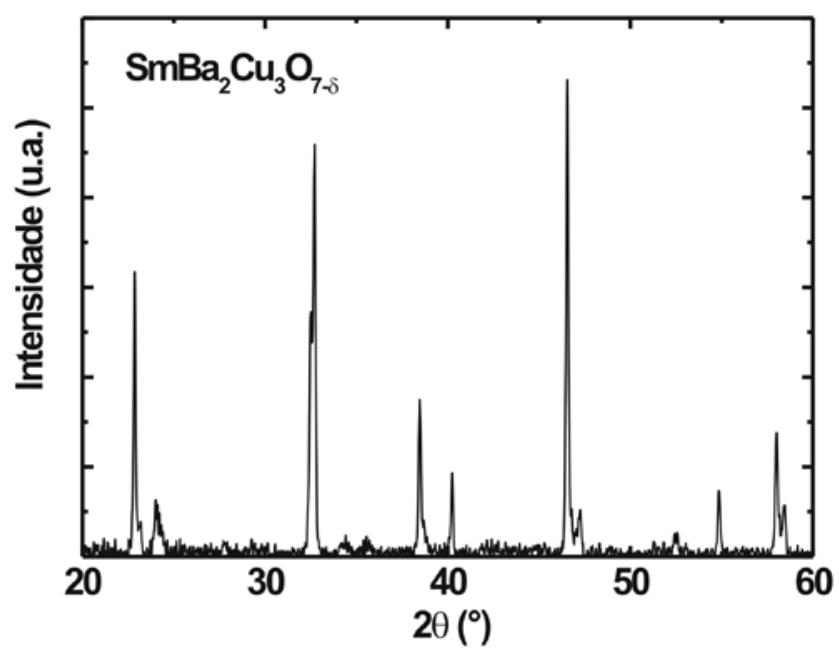

Figura 1: DRX da amostra de SBCO após a sinterização. [Figure 1: XRD of the SBCO sample after sintering.] magnéticas e elétricas com o tempo e a temperatura $[9,10]$, o qual reflete o ordenamento dos átomos de oxigênio. Uma das primeiras medidas direta da taxa de saltos e energia de ativação de oxigênio intersticial em SBCO foi realizada por nós, por intermédio de medidas de relaxação anelástica [11].

Neste trabalho, são apresentados resultados de espectroscopia anelástica obtidas em uma amostra de óxido supercondutor $\mathrm{SmBa}_{2} \mathrm{Cu}_{3} \mathrm{O}_{7-\delta}$, onde foi observado um processo de relaxação que foi atribuído à mobilidade de oxigênio intersticial.

\section{EXPERIMENTAL}

As amostras utilizadas neste trabalho foram preparadas pela mistura dos óxidos $\mathrm{Sm}_{2} \mathrm{O}_{3}, \mathrm{BaCO}_{3}$ e $\mathrm{CuO}$, numa proporção molar de 0,5:2:3. Os pós resultantes foram homogeneizados em uma almofariz de ágata e pastilhados com 0,5 GPa numa matriz retangular $\left(40 \times 5 \times 4 \mathrm{~mm}^{3}\right)$. As amostras foram sinterizadas quatro vezes com homogeneizações intermediárias. Os ciclos térmicos foram efetuados com taxa de aquecimento de $300 \mathrm{~K} / \mathrm{h}$ até 1293 $\mathrm{K}$, permanecendo nesta temperatura por $24 \mathrm{~h}$ e depois trazidas à temperatura ambiente numa taxa de $-60 \mathrm{~K} / \mathrm{h}$. Após a sinterização, os pós foram analisados por medidas de difração de raios X (DRX) e somente uma fase estava presente, conforme pode ser visto na Fig. 1. Para as medidas de espectroscopia anelástica, barras retangulares de 14 x 4 x $3 \mathrm{~mm}^{3}$ foram cortadas da pastilha de SBCO (densidade $\left.6,47 \mathrm{~g} / \mathrm{cm}^{3}\right)$.

O princípio de medida do atrito interno é muito simples: a amostra é colocada a vibrar em seu modo fundamental (vibrações livres ou forçadas), que causa a dissipação de energia sob a forma de calor, devido à existência de atrito interno. Essa dissipação de energia pode ser medida direta ou indiretamente. Em nosso caso, utilizamos um Pêndulo de Torção, onde a dissipação de energia por ciclo é medida através do decremento logarítmico que é, a menos de uma constante, o próprio atrito interno da amostra.

As medidas de espectroscopia anelástica (atrito interno), foram efetuadas utilizando-se um Pêndulo de Torção [12], com freqüência de oscilação em torno de 30 $\mathrm{Hz}$ e temperatura variando entre 77 e $700 \mathrm{~K}$.

Com o objetivo de variar a quantidade de oxigênio, a amostra foi submetida a um tratamento de desgaseificação (recozimento) em Ultra-Alto-Vácuo (UHV) a $900{ }^{\circ} \mathrm{C}$ por $75 \mathrm{~min}$.

\section{RESULTADOS E ANÁLISES}

A Fig. 2 mostra um espectro anelástico típico para a amostra de SBCO, medido com freqüência em torno de $40 \mathrm{~Hz}$. Podemos observar claramente a presença de uma estrutura de relaxação em torno de $500 \mathrm{~K}$ e um "degrau" na curva de freqüência. Estas são duas características marcantes 
de um processo de relaxação do tipo Debye. Para confirmar tal suposição, a amostra foi medida com outras duas freqüências, cujo resultado é mostrado na Fig. 3. Podemos observar, que há um deslocamento do pico para a região de alta temperatura, mostrando que o mesmo é termicamente ativado, confirmando a suposição que o pico em questão é devido a um processo de relaxação do tipo Debye $[5,11]$.

A amostra foi então submetida a um recozimento e submetida a uma nova série de medidas de espectroscopia anelástica. A Fig. 4 mostra o espectro anelástico para a amostra de SBCO, medida com freqüência em torno de 40 $\mathrm{Hz}$, da maneira como foi sinterizada e após o recozimento. Podemos observar a mesma estrutura de relaxação, porém, com uma intensidade menor, mostrando que houve perda de parte do oxigênio intersticial (o efeito da redução da

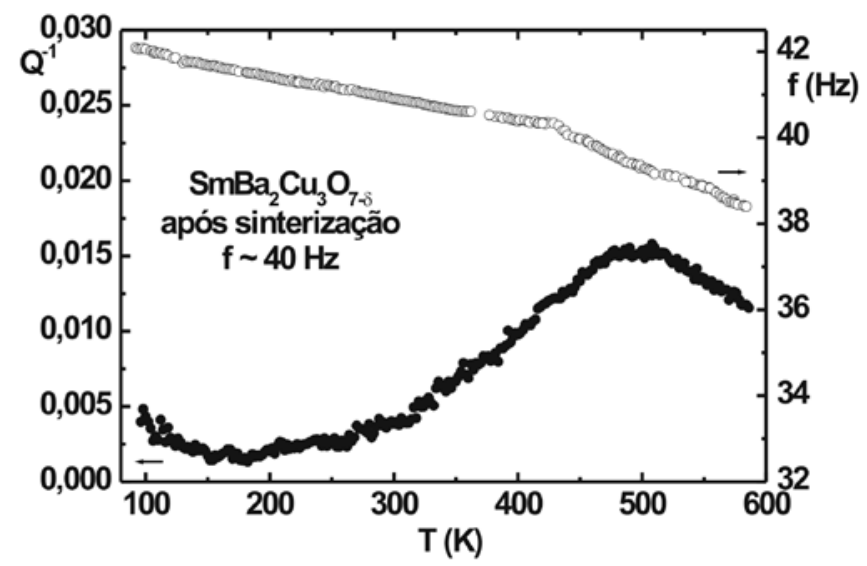

Figura 2: Atrito interno e freqüência para a amostra de SBCO medida com freqüência em torno de $40 \mathrm{~Hz}$.

[Figure 2: Internal friction and frequency for the SBCO sample, measured at a frequency around $40 \mathrm{~Hz}$.]

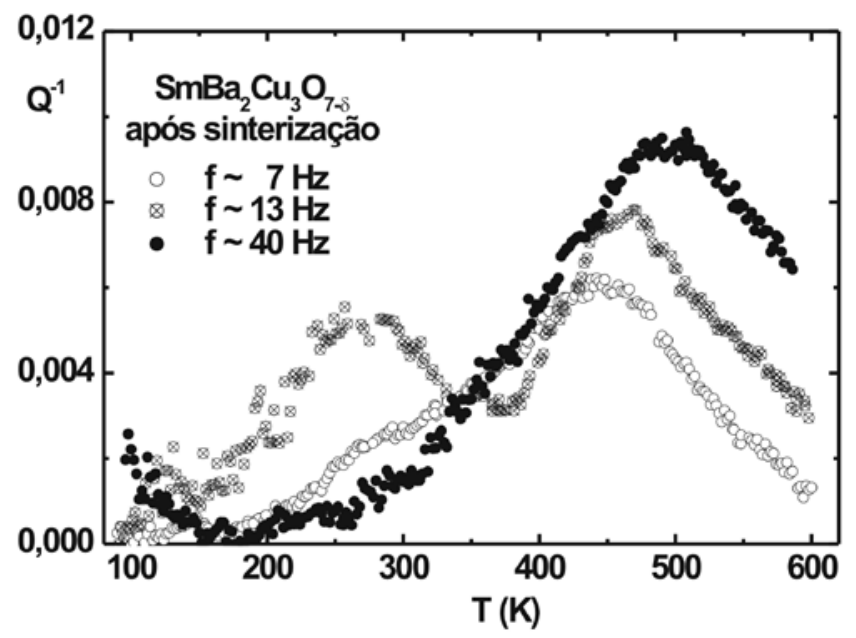

Figura 3: Atrito interno para a amostra de SBCO após a sinterização usando freqüências diferentes.

[Figure 3: Internal friction measurements for the as prepared SBCO sample using different frequencies.]

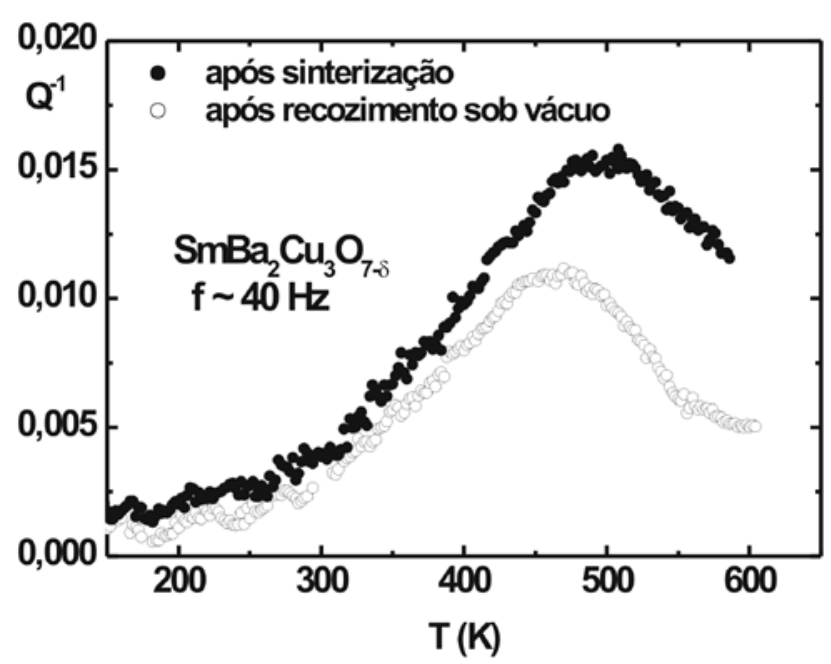

Figura 4: Comparação das medidas de atrito interno, efetuadas com freqüência de $40 \mathrm{~Hz}$.

[Figure 4: Comparative internal friction measurements taken at a frequency of $40 \mathrm{~Hz}$.]

quantidade de oxigênio é reduzir a intensidade do pico).

Processos de relaxação anelástica acima da temperatura ambiente foram previamente reportados para $\mathrm{RBa}_{2} \mathrm{Cu}_{3} \mathrm{O}_{7}$ $(\mathrm{R}=\mathrm{Y}, \mathrm{La}, \mathrm{Nd})[13,14]$ e para SBCO [11]. Tais efeitos tem energia de ativação da ordem de $1 \mathrm{eV}$ e são devidos à mobilidade de oxigênio nos planos basais.

Devido ao fato destes processos de relaxação ser observados para altas concentrações de oxigênio (quando o material é ortorrômbico), eles devem envolver saltos dentro ou fora dos planos $\mathrm{CuO}$ que formam a estrutura 123 e sua energia de ativação inclui a energia de ligação dos átomos de oxigênio nestes planos. Tais saltos constituem passos elementares da difusão de longo alcance de átomos de oxigênio nos planos $\mathrm{Cu}(1)-\mathrm{O}$ e de fato, ambos os processos, anelástico e difusão, tem energia de ativação comparáveis na fase ortorrômbica [14].

Neste sentido, nós propomos que este pico seja devido aos saltos dos átomos de oxigênio $\mathrm{O}(1)-\mathrm{O}(5)$ nos planos $\mathrm{Cu}-$ O na fase ortorrômbica [11].

\section{CONCLUSÕES}

Foram efetuadas medidas de espectroscopia anelástica em amostras do óxido SBCO utilizando a técnica do pêndulo de torção. Os resultados mostraram a presença de um pico de relaxação termicamente ativado, dependente da quantidade de oxigênio intersticial presente na amostra. Este pico foi atribuído aos saltos de átomos de oxigênio nas posições $\mathrm{O} 1$ e $\mathrm{O} 5$ da rede.

\section{AGRADECIMENTOS}

Os autores agradecem a Willians Govedise e Thiago Dorta Mariano pelo suporte técnico; ao Programa de 
Formação Continuada da Secretaria da Educação do Estado de S. Paulo, FAPESP, CNPq e FUNDUNESP pelo suporte financeiro.

\section{REFERÊNCIAS}

[1] M. Murakami, S. I. Yoo, T. Higuchi, N. Sakai, J. Weltz, N. Koshizuka, S. Tanaka, Jap. J. Appl. Phys. 33 (1994) L715. [2] S. I. Yoo, N. Sakai, H. Takaichi, T. Higuchi, M. Murakami, Appl. Phys. Lett. 65 (1994) 633.

[3] Ch. Klauns, M. Sumida, M. Tagami, Y. Yamada, Y. Shiorara, Z. Phys. B 96 (1994) 207.

[4] B. W. Veal, H. You, A. P. Paulikas, H. Shi, Y. Fang, J. W. Downey, Phys. Rev. B 42 (1990) 4770.

[5] A. S. Nowick, B. S. Berry, Anelastic Relaxation in Crystalline Solids, Academic Press, New York (1972).
[6] G. Cannelli, R. Cantelli, F. Cordero, F. Trequattrini, C. R. Grandini, M. Ferretti, Physica C 282 (1997) 1457.

[7] F. Cordero, C. R. Grandini, G. Cannelli, R. Cantelli, F. Trequattrini, M. Ferretti, Phys. Rev. B 57 (1998) 8580.

[8] F. Cordero, C. R. Grandini, R. Cantelli, Physica C 305 (1998) 251.

[9] J.-S. Zhou, H. Chen, J. B. Goodenough, Phys. Rev. B 50 (1994) 4168.

[10] F. C. Chou, D. C. Johnston, Phys. Rev. B 54 (1996) 572.

[11] C. R. Grandini, J. M. A. Gimenez, A. G. Cunha, Physica C 408 (2004) 741.

[12] C. R. Grandini, Rev. Bras. Aplic. Vácuo 21 (2002) 13 [13] J. L. Tallon, B.-E. Mellander, Science 258 (1992) 781. [14] G. Canelli, R. Cantelli, F. Cordero, F. Trequattrini, Solid State Comm. 77 (1991) 429. 